International Journal of Pure and Applied Mathematics

Volume 109 No. $3 \quad 2016,737-742$

ISSN: 1311-8080 (printed version); ISSN: 1314-3395 (on-line version)

url: http://www.ijpam.eu

doi: 10.12732/ijpam.v109i3.20

\title{
SOME RESULTS FOR CONTINUOUS FUNCTIONS ON WEAK SPACES
}

\author{
Young Key Kim ${ }^{1}$, Won Keun Min ${ }^{2}$ \\ ${ }^{1}$ Department of Mathematics \\ MyongJi University \\ Yongin, 449-728, KOREA \\ ${ }^{2}$ Department of Mathematics \\ Kangwon National University \\ Chuncheon, 200-701, KOREA
}

\begin{abstract}
In this article, we consider the triple $\left(X, w, \tau_{X}\right)$ where $w$ is a weak structure with an associated topology $\tau$. The purpose of this article is to study the relationships among several continuities( continuity, $W\left(W^{*}\right)$-continuity, $w O$-continuity, $w K$-continuity) defined in $w$-spaces with associated topologies.

In particular, we investigate the new continuity, say $O w$-continuity, defined in $w$-spaces with associated topologies, and study the characterizations of $O w$-continuity and the relationships among $O w$-continuity and several continuities.
\end{abstract}

AMS Subject Classification: 54A05, 54B10, 54C10, 54D30

Key Words: weak spaces, $W^{*}$-continuous, $W$-continuity, $W O$-continuity, $W K$-continuity, $w O$-continuity, $w K$-continuity, $O w$-continuity

\section{Introduction}

In [7], Siwiec introduced the notions of weak neighborhoods and weak base in a topological space. The author introduced the weak neighborhood systems

$\begin{array}{lr}\text { Received: } & \text { May 31, 2016 } \\ \text { Revised: } & \text { July 20, 2016 } \\ \text { Published: } & \text { September 30, 2016 }\end{array}$

(c) 2016 Academic Publications, Ltd. url: www.acadpubl.eu 
defined by using the notion of weak neighborhoods in [4]. The weak neighborhood system induces a weak neighborhood space (briefly WNS) which is independent of neighborhood spaces [2] and general topological spaces [1, 3]. We introduced the notion of weak structure [5] which is defined by the properties of new interior operator and closure operator in a WNS, and investigated the notions of $W$-continuity and $W^{*}$-continuity [5]. In [6], we introduced the notions of $W O$-continuity and $W K$-continuity on associated $w$-spaces. In particular, we investigate some properties and relationships between $W O$-continuity, $W K$ continuity, $W$-continuity, $W^{*}$-continuity and continuity on associated $w$-spaces.

In this paper, for a $w$-space $(X, w)$, a topology $\tau_{X}$ on $X$ is called an associated topology on $X$ if $w \subseteq \tau$. we will call the triple $\left(X, w, \tau_{X}\right)$ a $w$-space with an associated topology $\tau$. The purpose of this paper is to study the relationships among several continuities( continuity, $W\left(W^{*}\right)$-continuity, $w O$-continuity, $w K$-continuity) defined in $w$-spaces with associated topologies. In particular, we investigate the new continuity, say $O w$-continuity, defined in $w$-spaces with associated topologies, and study the characterizations of $O w$-continuity and the relationships among $\mathrm{Ow}$-continuity and several continuities.

\section{Preliminaries}

Definition $2.1([5])$. Let $X$ be a nonempty set. A subfamily $w_{X}$ of the power set $P(X)$ is called a weak structure on $X$ if it satisfies the following:

(1) $\emptyset \in w_{X}$ and $X \in w_{X}$.

(2) For $U_{1}, U_{2} \in w_{X}, U_{1} \cap U_{2} \in w_{X}$.

Then the pair $\left(X, w_{X}\right)$ is called a $w$-space on $X$. Then $V \in w_{X}$ is called a $w$-open set and the complement of a $w$-open set is a $w$-closed set.

The collection of all $w$-open sets (resp. $w$-closed sets) in a $w$-space $X$ will be denoted by $W O(X)$ (resp. $W C(X))$. We set $W(x)=\{U \in W O(X): x \in U\}$.

Definition $2.2([5])$. Let $\left(X, w_{X}\right)$ be a $w$-space. For a subset $A$ of $X$, the $w$-closureof $A$ and the w-interior [5] of $A$ are defined as the following:

(1) $w C l(A)=\cap\left\{F: A \subseteq F, X-F \in w_{X}\right\}$.

(2) $w \operatorname{Int}(A)=\cup\left\{U: U \subseteq A, U \in w_{X}\right\}$.

Theorem $2.3([5])$. Let $\left(X, w_{X}\right)$ be a $w$-space and $A \subseteq X$.

(1) If $A$ is $w$-open, then $w \operatorname{Int}(A)=A$.

(2) If $A$ is $w$-closed, then $w C l(A)=A$. 


\section{Main Results}

Definition 3.1. Let $X$ be a nonempty set and let $(X, w)$ be a $w$-space. A topology $\tau_{X}$ on $X$ is called an associated topology on $X$ if $w \subseteq \tau$. Then the triple $\left(X, w, \tau_{X}\right)$ is called a $w$-space with an associated topology $\tau$. The triple $\left(X, w, \tau_{X}\right)$ simply will be denoted by $\left(X, W_{\tau_{X}}\right)$.

Remark 3.2. Let $\left(X, w_{X}\right)$ be a $w$-space. Then clearly the family $\tau=$ $\{U \subset X: U=w \operatorname{Int}(U)\}$ is a topology and $w_{X} \subseteq \tau$ [5], and so it ia an associated topology on $X$ for a $w$-space $\left(X, w_{X}\right)$.

Definition 3.3. Let $f:\left(X, W_{\tau_{X}}\right) \rightarrow\left(Y, W_{\tau_{Y}}\right)$ be a function on $w$-spaces with two associated topologies $\tau_{X}$ and $\tau_{Y}$.

Then $f$ is said to be

(1) wO-continuous if for $x \in X$ and for each $\tau_{Y}$-open set $V$ containing $f(x)$, there is a $w$-open set $U$ containing $x$ such that $f(U) \subseteq V$,

(2) wK-continuous if for every $\tau_{Y}$-open set $V$ in $Y, f^{-1}(V)$ is a $w$-open set in $X$.

(3) $W$-continuous [5] if for $x \in X$ and $V \in W(f(x))$, there is $U \in W(x)$ such that $f(U) \subset V$.

(4) $W^{*}$-continuous [5] if for every $A \in W(f(x)), f^{-1}(A)$ is in $W(x)$.

Remark 3.4. For a function $f:\left(X, W_{\tau_{X}}\right) \rightarrow\left(Y, W_{\tau_{Y}}\right)$, from Definition 3.3 and the next examples, we get the following implications:

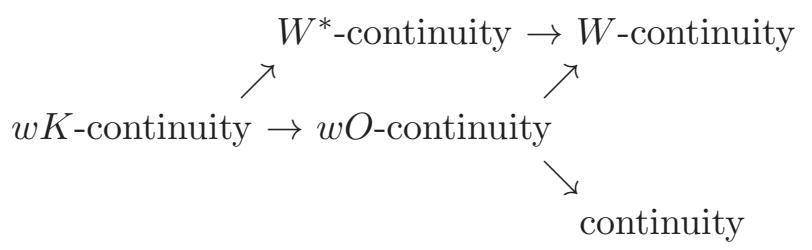

Example 3.5. For $X=\{a, b, c\}$, let $w=\{\emptyset,\{a\},\{b\}, X\}$ be a $w$-structure.

(1) Let an associated topology $\tau=\{\emptyset,\{a\},\{b\},\{a, b\},\{a, c\}, X\}$. Consider the identity function $f:\left(X, W_{\tau_{X}}\right) \rightarrow\left(X, W_{\tau_{X}}\right)$. Then $f$ is continuous, $W^{*_{-}}$ continuous and $W$-continuous but neither $w O$-continuous nor $w K$-continuous.

(2) Consider $\mu=\{\emptyset,\{a\},\{b\},\{a, b\}, X\}$ and the identity function $f:\left(X, W_{\mu_{X}}\right) \rightarrow$ $\left(X, W_{\tau_{X}}\right)$. Then $f$ is $W$-continuous but not continuous.

(3) Consider a function $f:\left(X, W_{\tau_{X}}\right) \rightarrow\left(X, W_{\tau_{X}}\right)$ defined as $f(b)=$ $a ; f(a)=f(c)=b$. Then $f$ is continuous but not $W$-continuous.

(4) For an associated topology $\mu=\{\emptyset,\{a\},\{b\},\{a, b\}, X\}$, consider the identity function $f:\left(X, W_{\mu_{X}}\right) \rightarrow\left(X, W_{\mu_{X}}\right)$. Then $f$ is $w O$-continuous but not 
$w K$-continuous.

Remark 3.6. Let $X$ be a nonempty set and let $(X, \tau)$ be a topological space. A subfamily $w_{\tau}$ of the power set $P(X)$ is called an associated weak structure [6] on $X$ if $\tau \subseteq w_{\tau}$.

Let $f:\left(X, w_{\tau}\right) \rightarrow(Y, \mu)$ be a function on an associated $w$-space with $\tau$ and a topological space $(Y, \mu)$. Then $f$ is said to be

(1) WO-continuous [6] if for $x \in X$ and $V \in O(f(x))$, there is $U \in W(x)$ such that $f(U) \subseteq V$,

(2) WK-continuous [6] if for every open set $V$ in $Y, f^{-1}(V)$ is a $w$-open set in $X$.

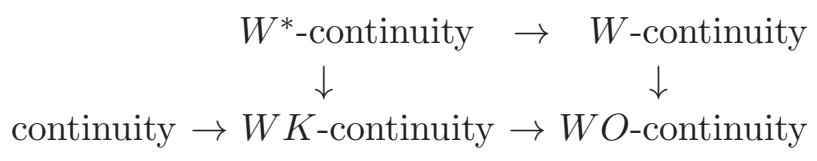

Theorem 3.7. Let $f:\left(X, W_{\tau_{X}}\right) \rightarrow\left(Y, W_{\tau_{Y}}\right)$ be a function. Then the following statements are equivalent:

(1) $f$ is $w O$-continuous.

(2) $f(w C l(A)) \subseteq \operatorname{cl}(f(A))$ for $A \subseteq X$.

(3) $w C l\left(f^{-1}(V)\right) \subseteq f^{-1}(\operatorname{cl}(V))$ for $V \subseteq Y$.

(4) $f^{-1}(\operatorname{int}(V)) \subseteq w \operatorname{Int}\left(f^{-1}(V)\right)$ for $V \subseteq Y$

Proof. Obvious.

Theorem 3.8. Let $f:\left(X, W_{\tau_{X}}\right) \rightarrow\left(Y, W_{\tau_{Y}}\right)$ be a function.

(1) Then the following are equivalent:

(i) $f$ is $w K$-continuous.

(ii) For every closed set $F$ in $Y, f^{-1}(F)$ is $w$-closed in $X$.

(2) If $f$ is $w K$-continuous, then $w O$-continuous.

Proof. Obvious.

Now, we define a new notion of continuous function between an associated $w$-spaces with two associated topologies.

Definition 3.9. Let $f:\left(X, W_{\tau_{X}}\right) \rightarrow\left(Y, W_{\tau_{Y}}\right)$ be a function. The function $f$ is said to be $O w$-continuous if for each $w$-open set $V$ containing $f(x)$, there is a $\tau_{w_{X}}$-open set $U$ containing $x$ such that $f(U) \subseteq V$.

Obviously, it is that every $W$-continuous and every continuous function are $O w$-continuous but the converses may not be true as the next examples: 
Example 3.10. (1) As Example 3.5, for $X=\{a, b, c\}$ and a $w$-structure $w=\{\emptyset,\{a\},\{b\}, X\}$, let an associated topology $\nu=\{\emptyset,\{a\},\{b\},\{a, b\},\{b, c\}, X\}$. Consider a function $f:\left(X, W_{\nu_{X}}\right) \rightarrow\left(X, W_{\nu_{X}}\right)$ defined as $f(a)=a ; f(b)=$ $f(c)=b$. Then $f$ is $O w$-continuous but not $W$-continuous.

(2) In the above (1), consider $\mu=\{\emptyset,\{a\},\{b\},\{a, b\}, X\}$ and the function $f:\left(X, W_{\mu_{X}}\right) \rightarrow\left(X, W_{\nu_{X}}\right)$. Then $f$ is $O w$-continuous but not continuous.

Remark 3.11. Let $f:\left(X, W_{\tau_{X}}\right) \rightarrow\left(Y, W_{\tau_{Y}}\right)$ be a function. Then we get the following implications:

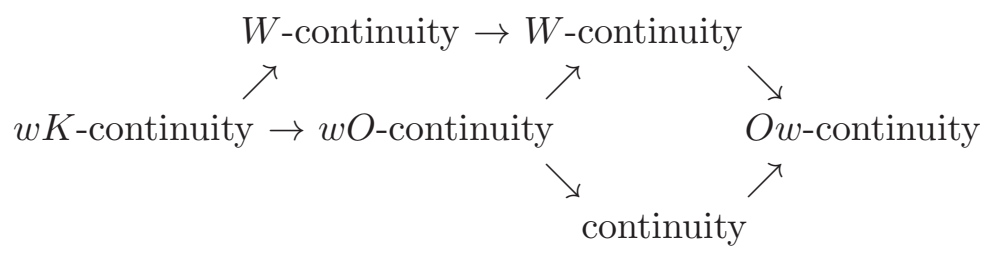

Theorem 3.12. Let $f:\left(X, W_{\tau_{X}}\right) \rightarrow\left(Y, W_{\tau_{Y}}\right)$ be a function. Then the following statements are equivalent:

(1) $f$ is $O w$-continuous.

(2) $f(c l(A)) \subseteq w C l(f(A))$ for $A \subseteq X$.

(3) $\operatorname{cl}\left(f^{-1}(B)\right) \subseteq f^{-1}(w C l(B))$ for $B \subseteq Y$.

(4) $f^{-1}(w \operatorname{Int}(V)) \subseteq \operatorname{int}\left(f^{-1}(V)\right)$ for $V \subseteq Y$.

(5) If for every $w$-open set $V$ in $Y, f^{-1}(V)$ is an open set in $X$.

Proof. $(1) \Rightarrow(2)$ For $x \in \operatorname{cl}(A)$, assume that $f(x)$ is not in $w C l(f(A))$. Then there exists a $w$-open set $V$ containing $f(x)$ such that $V \cap f(A)=\emptyset$. Вy $O w$-continuity, there is an open set $U$ containing $x$ such that $f(U) \subseteq V$, and it implies that $f(U) \cap f(A)=\emptyset$ and $U \cap A=\emptyset$. So it is a contradiction and we have $f(c l(A)) \subseteq w C l(f(A))$.

$(2) \Rightarrow(3)$ Let $A=f^{-1}(B)$ for $B \subseteq Y$. Then by hypothesis, $f(\operatorname{cl}(A)) \subseteq$ $w C l(f(A))=w C l\left(f\left(f^{-1}(B)\right)\right) \subseteq w C l(B)$. Thus $c l\left(f^{-1}(B)\right) \subseteq f^{-1}(w C l(B))$.

$(3) \Rightarrow(4)$ It is obvious.

$(4) \Rightarrow(5)$ Let $V$ be a $w$-open set $V$ in $Y$. Then $V=w \operatorname{Int}(V)$ and by hypothesis, $f^{-1}(V)=f^{-1}(w \operatorname{Int}(V)) \subseteq \operatorname{int}\left(f^{-1}(V)\right)$, and so $f^{-1}(V)$ is open in $X$.

$(5) \Rightarrow(1)$ For $x \in X$, let $V$ be any $w$-open set containing $f(x)$. Then by (5), it follows that $f^{-1}(V)$ is an open set containing $x$ and $x \in f^{-1}(V) \subseteq$ $\operatorname{int}\left(f^{-1}(V)\right)$. From this fact, there exists an open set $U$ such that $x \in U \subseteq$ $f^{-1}(V)$. It implies that $f$ is $O w$-continuous. 
We recall Remark 3.2: Let $(X, w)$ be a $w$-space. Then $\tau_{X}=\{U \subset X: U=$ $w \operatorname{Int}(U)\}$ is an associated topology such that $w \subseteq \tau_{X}$.

Theorem 3.13. Let $f:\left(X, W_{\tau_{X}}\right) \rightarrow\left(Y, W_{\tau_{Y}}\right)$ be a function. If the two associate topologies $\tau_{X}=\{U \subset X: U=w \operatorname{Int}(U)\}$ and $\tau_{Y}=\{U \subset Y: U=$ $w \operatorname{Int}(U)\}$, then we get the following things:

(1) $w O$-continuity $=$ continuity:

(2) $W$-continuity $=O w$-continuity:

Proof. (1) Let $f:\left(X, W_{\tau_{X}}\right) \rightarrow\left(Y, W_{\tau_{Y}}\right)$ be a function. From Remark 3.11, it is sufficient to show that the continuity implies $w O$-continuity. Suppose that $f$ is continuous. For $x \in X$, let $V$ be any $\tau_{Y}$-open set containing $f(x)$. By continuity of $f$, there is an open set $U$ containing $x$ such that $f(U) \subseteq V$, and since $\tau_{X}=\{U \subset X: U=w \operatorname{Int}(U)\}$, there exists a $w$-open set $U_{x}$ containing $x$ such that $U_{x} \subseteq U$. It implies $f$ is $w O$-continuous.

(2) It is similar to the proof of (1).

In summary, from the above theorem, the following diagram is obtained:

Remark 3.14. Let $f:\left(X, W_{\tau_{X}}\right) \rightarrow\left(Y, W_{\tau_{Y}}\right)$ be a function. For the two associate topologies $\tau_{X}=\{U \subset X: U=w \operatorname{Int}(U)\}$ and $\tau_{Y}=\{U \subset Y: U=$ $w \operatorname{Int}(U)\}$, the following diagram is obtained:

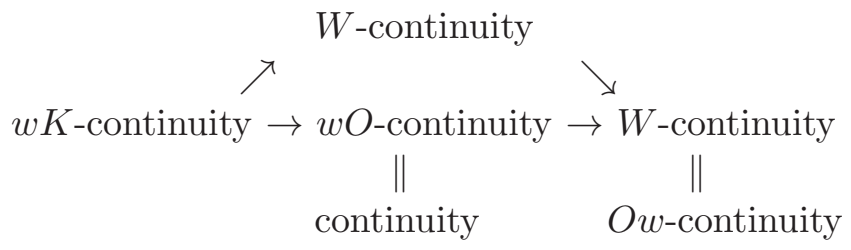

\section{Acknowledgments}

The authors thank the anonymous referees for their valuable suggestions in improving this paper. This study was supported by 2015 Research Grant from Kangwon National University(No. D1000413-01-01)

\section{References}

[1] Á. Csázár; Generalized Topology, Generalized Continuity, Acta Math. Hungar., 96 (2002), 351-357. 
[2] D. C. Kent and W. K. Min; Neighborhood Spaces, International Journal of Mathematics and Mathematical Sciences, 32(7) (2002), 387-399.

[3] W. K. Min; Some Results on Generalized Topological Spaces and Generalized Systems, Acta Math. Hungar., 108 (1-2) (2005), 171-181.

[4] W. K. Min; On Weak Neighborhood Systems and Weak Neighborhood Spaces, Acta Math. Hungar., (2008) DOI:10.1007/s10474-008-7205-8.

[5] W. K. Min and Y. K. Kim; On Weak Structures and w-spaces, Far East Journal of Mathematical Sciences, 97(5) (2015), 549-561.

[6] W. K. Min and Y. K. Kim; $W O$-continuity and $W K$-continuity On Associated $w$-spaces, International Journal of Pure and Applied Mathematics, 102(2) (2015), 349-356.

[7] F. Siwiec; On Defining a Space by a Weak Base, Pacific Journal of Mathematics, 52(1) (1974), 351-357. 
